\title{
Fast and Optimal Sequence-to-Graph Alignment Guided by Seeds
}

\author{
Pesho Ivanov, Benjamin Bichsel, and Martin Vechev \\ Department of Computer Science, \\ ETH Zurich, Switzerland \\ \{firstname. lastname\}@inf.ethz.ch
}

\begin{abstract}
We present a novel $\mathrm{A}^{\star}$ seed heuristic enabling fast and optimal sequence-to-graph alignment, guaranteed to minimize the edit distance of the alignment assuming non-negative edit costs. We phrase optimal alignment as a shortest path problem and solve it by instantiating the $\mathrm{A}^{\star}$ algorithm with our novel seed heuristic. The key idea of the seed heuristic is to extract seeds from the read, locate them in the reference, mark preceding reference positions by crumbs, and use the crumbs to direct the $\mathrm{A}^{\star}$ search. We prove admissibility of the seed heuristic, thus guaranteeing alignment optimality.

Our implementation extends the free and open source ASTARIX aligner and demonstrates that the seed heuristic outperforms all state-of-the-art optimal aligners including GraphAligner, VArgas, PASGAL, and the prefix heuristic previously employed by ASTARIX. Specifically, we achieve a consistent speedup of $>60 \times$ on both short Illumina reads and long HiFi reads (up to 25kbp), on both the E. coli linear reference genome (1Mbp) and the $\mathrm{MHC}$ variant graph (5Mbp). Our speedup is enabled by the seed heuristic consistently skipping $>99.99 \%$ of the table cells that optimal aligners based on dynamic programming compute.
\end{abstract}

Keywords: Next-generation sequencing $\cdot$ Optimal alignment $\cdot$ Genome graph $\cdot$ Shortest path $\cdot A^{\star}$ algorithm - Long reads · Seeding

\section{Introduction}

Alignment of reads to a reference genome is an essential and early step in most bioinformatics pipelines. While linear references have been used traditionally, an increasing interest is directed towards graph references capable of representing biological variation [1]. Specifically, a sequence-to-graph alignment is a base-to-base correspondence between a given read and a walk in the graph. As sequencing errors and biological variation result in inexact read alignments, edit distance is the most common metric that alignment algorithms optimize in order to find the most probable read origin in the reference.

Suboptimal Alignment. The most popular paradigm in read aligning, seed-and-extend [2-4], locates substrings of the read (called seeds) in the reference, and extends these matches to align the full read.

While such a heuristic may produce acceptable alignments in many cases, it fundamentally does not provide quality guarantees, resulting in suboptimal alignment accuracy.

Optimal Alignment: Key Challenges. In contrast, finding optimal alignments is desirable but expensive in the worst case, requiring $\mathcal{O}(\mathrm{Nm})$ time [5], for graph size $N$ and read length $m$. Unfortunately, most optimal sequence-to-graph aligners rely on dynamic programming (DP) and always reach this worst-case asymptotic runtime. Such aligners include Vargas [6], PaSGAL [7], GraphAligner [8], HGA [9], VG [1], which use bit-level optimizations and parallelization to increase their throughput.

In contrast, ASTARIX [10] follows the promising direction of using a heuristic to avoid worst-case runtime on realistic data. To this end, ASTARIX rephrases the task of alignment as a shortest-path problem in an alignment graph extended by a trie index, and solves it using the $\mathrm{A}^{\star}$ algorithm instantiated with a problemspecific prefix heuristic. Importantly, its choice of heuristic only affects performance, not optimality. Unlike DP-based algorithms, this prefix heuristic allows scaling sublinearly with the reference size, substantially increasing performance on large genomes. However, it can only efficiently align reads of limited length. 
This Work: Optimal Alignment for Short and Long Reads. In this work, we address the key challenge of scaling to long HiFi reads, while retaining the superior scaling of ASTARIX in the size of the reference graph. To this end, we instantiate the $\mathrm{A}^{\star}$ algorithm with a novel seed heuristic, which outperforms existing optimal aligners on both short and long HiFi reads. Specifically, the seed heuristic utilizes information from the whole read to narrowly direct the $\mathrm{A}^{\star}$ search by placing crumbs on graph nodes which lead up to a seed match, i.e., an exact match of a substring of the read.

Overall, the contributions presented in this work are:

1. A novel $\mathrm{A}^{\star}$ seed heuristic that exploits information from the whole read to quickly align it to a general graphs reference.

2. An optimality proof showing that the seed heuristic always finds an alignment with minimal edit distance.

3. An implementation of the seed heuristic as part of the ASTARIX aligner.

4. An extensive evaluation of our approach, showing that we align both short Illumina reads and long HiFi reads to both linear and graph references $\geq 60 \times$ faster than existing optimal aligners.

5. A demonstration of superior empirical runtime scaling in the reference size $N: N^{0.46}$ on Illumina reads and $N^{0.11}$ on HiFi reads.

\section{Prerequisites}

We define the task of alignment as a shortest path problem (§2.1) to be solved using the $\mathrm{A}^{\star}$ algorithm (§2.2).

\subsection{Problem Statement: Alignment as Shortest Path}

In the following, we formalize the task of optimally aligning a read to a reference graph in terms of finding a shortest path in an alignment graph. Our discussion closely follows [10, §2] and is in line with [11].

Reference Graph. A reference graph $G_{\mathrm{r}}=\left(V_{\mathrm{r}}, E_{\mathrm{r}}\right)$ encodes a collection of references to be considered when aligning a read. Its directed edges $E_{\mathrm{r}} \subseteq V_{\mathrm{r}} \times V_{\mathrm{r}} \times \Sigma$ are labeled by nucleotide letters from $\Sigma=\{\mathrm{A}, \mathrm{C}, \mathrm{G}, \mathrm{T}\}$, hence any walk $\pi_{\mathrm{r}}$ in $G_{\mathrm{r}}$ spells a string $\sigma\left(\pi_{\mathrm{r}}\right) \in \Sigma^{*}$.

An alignment of a read $q \in \Sigma^{*}$ to a reference graph $G_{\mathrm{r}}$ consists of (i) a walk $\pi_{\mathrm{r}}$ in $G_{\mathrm{r}}$ and (ii) a sequence of edits (matches, substitutions, deletions, and insertions) that transform $\sigma\left(\pi_{r}\right)$ to $q$. An alignment is optimal if it minimizes the sum of edit costs for a given real-valued cost model $\Delta=\left(\Delta_{\text {match }}, \Delta_{\text {subst }}, \Delta_{\text {del }}, \Delta_{\text {ins }}\right)$. Throughout this work, we assume that edit costs are non-negative - a pre-requisite for the correctness of $\mathrm{A}^{\star}$. Further, we assume that $\Delta_{\text {match }} \leq \Delta_{\text {subst }}, \Delta_{\text {ins }}, \Delta_{\text {del }}$-a prerequisite for the correctness of our heuristic.

We note that our approach naturally works for cyclic reference graphs.

Alignment Graph. In order to formalize optimal alignment as a shortest path finding problem, we rely on an alignment graph $G_{\mathrm{a}}^{q}=\left(V_{\mathrm{a}}^{q}, E_{\mathrm{a}}^{q}\right)$. Its nodes $V_{\mathrm{a}}^{q}$ are states of the form $\langle v, i\rangle$, where $v \in V_{\mathrm{r}}$ is a node in the reference graph and $i \in\{0, \ldots,|q|\}$ corresponds to a position in the read $q$. Its edges $E_{\mathrm{a}}^{q}$ are selected such that any path $\pi_{\mathrm{a}}$ in $G_{\mathrm{a}}^{q}$ from $\langle u, 0\rangle$ to $\langle v, i\rangle$ corresponds to an alignment of the first $i$ letters of $q$ to $G_{\mathrm{r}}$. Further, the edges are weighted, which allows us to define an optimal alignment of a read $q \in \Sigma^{*}$ as a shortest path $\pi_{\mathrm{a}}$ in $G_{\mathrm{a}}^{q}$ from $\langle u, 0\rangle$ to $\langle v,|q|\rangle$, for any $u, v \in V_{\mathrm{r}}$. Formally, the edges $E_{\mathrm{a}}^{q} \subseteq V_{\mathrm{a}}^{q} \times V_{\mathrm{a}}^{q} \times \Sigma_{\varepsilon} \times \mathbb{R}_{\geq 0}$ are defined as

$$
\begin{array}{llr}
\left(\langle u, i\rangle,\langle v, i+1\rangle, q[i], \Delta_{\text {match }}\right) \in E_{\mathrm{a}}^{q} & \text { if }(u, v, \ell) \in E_{\mathrm{r}}, \ell=q[i] & \text { (match) } \\
\left(\langle u, i\rangle,\langle v, i+1\rangle, q[i], \Delta_{\text {subst }}\right) \in E_{\mathrm{a}}^{q} & \text { if }(u, v, \ell) \in E_{\mathrm{r}}, \ell \neq q[i] & \text { (substitution) } \\
\left(\langle u, i\rangle,\langle v, i \quad\rangle, \varepsilon, \Delta_{\text {del }} \quad\right) \in E_{\mathrm{a}}^{q} & \text { if }(u, v, \ell) \in E_{\mathrm{r}} & \text { (deletion) } \\
\left(\langle u, i\rangle,\langle u, i+1\rangle, q[i], \Delta_{\text {ins }} \quad\right) \in E_{\mathrm{a}}^{q} & & \text { (insertion), }
\end{array}
$$

where $u, v \in V_{\mathrm{r}}, 0 \leq i<|q|, \ell \in \Sigma$, and $\varepsilon$ represents the empty string, indicating that letter $\ell$ was deleted. 


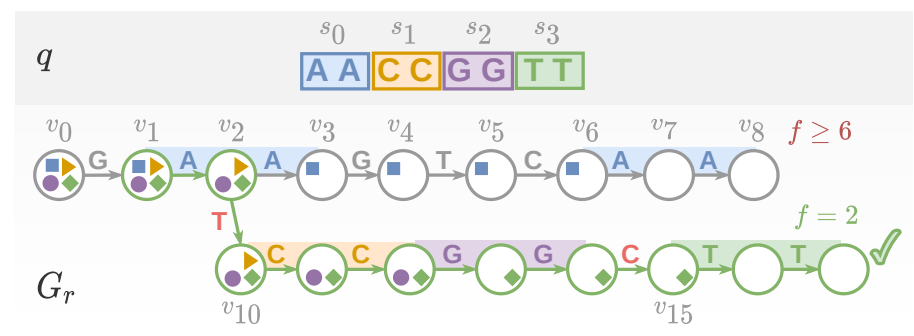

Fig. 1: A toy overview example using the seed heuristic to align a read $q$ to a reference graph $G_{\mathrm{r}}$. The read is split into four colored seeds, where their corresponding crumbs are shown inside reference graph nodes as symbols with matching color. The optimal alignment is highlighted as a green path ending with a tick $(\Omega)$ and includes one substitution $(\mathrm{T} \rightarrow \mathrm{A})$ and one deletion $(\mathrm{C})$.

\section{$2.2 \quad A^{\star}$ Algorithm for Finding a Shortest Path}

Given a weighted graph $G=(V, E)$, the $\mathrm{A}^{\star}$ algorithm finds a shortest path from sources $S \subseteq V$ to targets $T \subseteq V$. To prioritize paths that lead to a target, it relies on a heuristic function $h: V \rightarrow \mathbb{R}_{\geq 0}$, where $h(v)$ estimates the remaining length of the shortest path from a given node $v \in V$ to any target $t \in T$.

Algorithm. In a nutshell, the $\mathrm{A}^{\star}$ algorithm maintains a set of explored nodes, initialized by all possible starting nodes $S$. It then iteratively expands the explored state $v$ with lowest estimated total cost $f(v)$ by exploring all its neighbors. Here, $f(v):=g(v)+h(v)$, where $g(v)$ is the distance from $s \in S$ to $v$, and $h(v)$ is the estimated distance from $v$ to $t \in T$. When the $\mathrm{A}^{\star}$ algorithm expands a target node $t \in T$, it reconstructs the path leading to $t$ and returns it. For completeness, App. A.1 provides an implementation of $\mathrm{A}^{\star}$.

Admissible Heuristic. The $\mathrm{A}^{\star}$ algorithm is guaranteed to find a shortest path if its heuristic $h$ provides a lower bound on the distance to the closest target, often referred to as $h$ being admissible or optimistic.

Further, the performance of the $\mathrm{A}^{\star}$ algorithm relies critically on the choice of $h$. Specifically, it is crucial to have low estimates for the optimal paths but also to have high estimates for suboptimal paths.

Discussion. To summarize, we use the $\mathrm{A}^{\star}$ algorithm to find a shortest path from $\langle u, 0\rangle$ to $\langle v,|q|\rangle$ in $G_{a}^{q}$. To guarantee optimality, its heuristic function $h\langle v, i\rangle$ must provide a lower bound on the shortest distance from state $\langle v, i\rangle$ to a terminal state of the form $\langle w,|q|\rangle$. Equivalently, $h\langle v, i\rangle$ should lower bound the minimal cost of aligning $q[i:]$ to $G_{\mathrm{r}}$ starting from $v$, where $q[i$ :] denotes the suffix of $q$ starting at position $i$ (0-indexed). The key challenge is thus finding a heuristic that is not only admissible but also yields favorable performance.

\section{Seed Heuristic}

We instantiate the $\mathrm{A}^{\star}$ algorithm with a novel, domain-specific seed heuristic which allows to quickly align reads to a general reference graph. We first showcase our heuristic on an overview example (§3.1). Then, we formally define the heuristic and prove its admissibility (§3.2). Finally, we adapt our approach to rely on a trie, which leads to a critical speedup $(\S 3.3)$.

\subsection{Overview}

Fig. 1 showcases the seed heuristic on an overview example. It shows a read $q$ to be aligned to a reference graph $G_{\mathrm{r}}$. Our goal is to find an optimal alignment starting from an arbitrary node $v \in G_{\mathrm{r}}$. For simplicity of the exposition, we assume unit edit costs $\Delta=(0,1,1,1)$, which we will later generalize in $§ 3.2$.

Intuition. The intuition underlying the seed heuristic is similar to how Hansel and Gretel place breadcrumbs to find their trail back home. An optimal alignment can be found much faster if we place crumbs on nodes in the reference graph which lead towards more promising alignments. More specifically, a crumb in node $v$ indicates that a specific seed (a substring of $q$ ) can potentially be aligned exactly when the alignment 

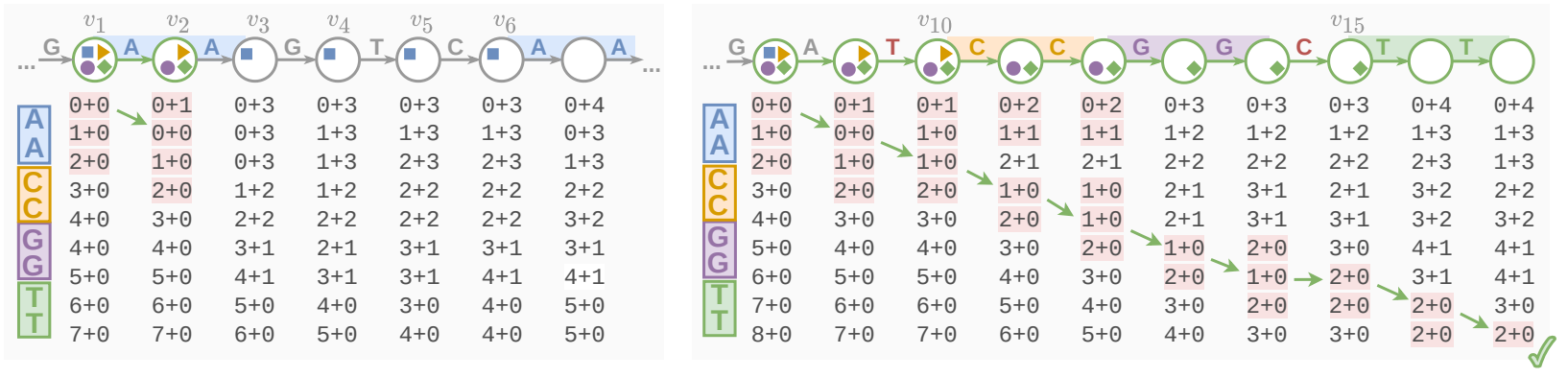

Fig. 2: Exploration of $G_{a}^{q}$, searching for a shortest path from the first to the last row using the seed heuristic. The table entry in the $i^{\text {th }}$ row (zero indexed) below node $v$ shows $g\langle v, i\rangle+h\langle v, i\rangle$, where $g\langle v, i\rangle$ is the shortest distance from any starting state $\langle u, 0\rangle$ to $\langle v, i\rangle$. States that may ${ }^{1}$ be expanded by the $\mathrm{A}^{\star}$ algorithm are highlighted in pink, and the rest of the states are shown for completeness even though they are never expanded. The shortest path corresponding to the best alignment is shown with green arrows $(\rightarrow)$.

proceeds through $v$. Fig. 1 shows these seeds as 4 colored sections of length 2 each, and represents their corresponding crumbs as $\square, \downarrow, \bigcirc$ and $\diamond$, respectively.

Seed Heuristic based on Crumbs. Based on the concept of crumbs, we further define our seed heuristic $h\langle v, i\rangle$ to be a lower bound on the cost for aligning $q[i:]$ starting from $v$. Clearly, all seeds occurring in $q[i:]$ must be aligned. For all such seeds, we expect to see their corresponding crumbs in node $v$. If a crumb of an expected seed is missing in node $v$, its corresponding seed $s$ could not possibly be aligned exactly, which will incur the cost of at least one substitution, insertion, or deletion. Assuming unit edit costs, $h\langle v, i\rangle$ is then simply the number of missing expected crumbs in $v$.

For example, in Fig. 1, four crumbs are expected if we start at $v_{2}$, but $\square$ is missing, so $h\left\langle v_{2}, 0\right\rangle=1$. Analogously, if we reach $v_{2}$ after aligning one letter from the read, we expect 3 crumbs (except $\square$ ), and we find them all in $v_{2}$, so $h\left\langle v_{2}, 1\right\rangle=0$.

Crumbs Precomputation. To precompute the crumbs for each seed, we first find all positions in $G_{\mathrm{r}}$ from which the seed aligns exactly. Fig. 1 shows these exact matches as colored sections of $G_{\mathrm{r}}$. Then, from each match we traverse $G_{\mathrm{r}}$ backwards and add crumbs to nodes that could lead to these matches. For example, because seed CC can be matched starting in node $v_{10}$, crumbs $>$ are placed on all nodes leading up to $v_{10}$. Similarly, seed AA has two exact matches, one starting in node $v_{0}$ and one starting in node $v_{6}$. However, we only add crumbs to nodes $v_{0}, v_{1}$, and $v_{3}-v_{6}$, but not to node $v_{2}$. This is because $v_{2}$ is (i) strictly after the beginning of the match of AA at $v_{1}$ and (ii) too far before the match of AA at $v_{6}$. Specifically, any alignment starting from node $v_{2}$ and still matching $\mathrm{AA}$ at $v_{6}$ would induce an overall cost of 4 (it would require deleting the 4 letters $A, G, T$, and $C$ ). Even without a crumb $\square$ on $v_{2}$, our heuristic provides a lower bound on the cost of such an alignment: it never estimates a cost of more than 4 , as discussed in $\S 3.2$.

Guiding the Search. Fig. 2 demonstrates how $h\langle v, i\rangle$ guides the $\mathrm{A}^{\star}$ algorithm towards the shortest path by showing which states may be expanded when using the seed heuristic. Specifically, the unique optimal alignment in Fig. 1 starts from node $v_{1}$, continues to $v_{2}$, and then proceeds through node $v_{10}$ (instead of $v_{3}$ ).

While the seed heuristic initially explores all states of the form $\langle v, 0\rangle$ (we discuss in $\S 3.3$ how to avoid this by using a trie), it skips expanding any state that involves nodes $v_{3}-v_{8}$. This improvement is possible because all these explored states are penalized by the seed heuristic by at least 3, while the shortest path of cost 2 will be found before considering states on nodes $v_{3}-v_{8}$. Here, the heuristic function accurately predicts that expanding $v_{10}$ may eventually lead to an exact alignment of seeds CC, GG and TT, while expanding $v_{3}$ may not lead to an alignment of either seed. In particular, the seed heuristic is not misled by the short-term benefit of correctly matching $A$ in $v_{2}$, and instead provides a long-term recommendation based on the whole

\footnotetext{
${ }^{1}$ Depending on how the $\mathrm{A}^{\star}$ algorithm handles tie-braking, different sets of states could be explored. For simplicity, we show all states that could potentially be explored.
} 
read. Thus, even though the walk to $v_{3}$ aligns exactly the first two letters of $q$, $\mathrm{A}^{\star}$ does not expand $v_{3}$ because the seed heuristic guarantees that the future cost will be at least 3 .

\subsection{Formal Definition}

Next, we formally define the seed heuristic function $h\langle v, i\rangle$. Overall, we want to ensure that $h\langle v, i\rangle$ is admissible, i.e., that it is a lower bound on the cost of a shortest path from $\langle v, i\rangle$ to some $\langle w,|q|\rangle$ in $G_{\mathrm{a}}^{q}$.

Seeds. We split read $q \in \Sigma^{*}$ into a set Seeds of non-overlapping seeds $s_{0}, \ldots, s_{\mid \text {Seeds } \mid-1} \in \Sigma^{*}$. For simplicity, in this work we ensure that all seeds have the same length and are consecutive, i.e., we split $q$ into substrings $s_{0} \cdot s_{1} \cdots s_{\mid \text {Seeds } \mid-1} \cdot t$, where all $s_{j}$ are seeds of length $l$ and we ignore the suffix $t$ of $q$, which is shorter than $l$. In this case, $k=\lfloor|q| / l\rfloor$. However, we note that our approach can be trivially generalized to seeds of different lengths or non-consecutive seeds as long as they do not overlap.

Matches. For each seed $s \in$ Seeds, we locate all nodes $u \in M(s)$ in the reference graph that can be the start of an exact match of $s$ :

$$
M(s):=\left\{u \in V_{\mathrm{r}} \mid \exists \text { walk } \pi \text { starting from } u \in G_{\mathrm{r}} \text { and spelling } \sigma(\pi)=s\right\} .
$$

To compute $M(s)$ efficiently, we leverage the trie introduced in $\S 3.3$.

Crumbs. For seed $s_{j}$ starting at position $i$ in $q$, we place crumbs on all nodes $u \in V_{\mathrm{r}}$ which can reach a node $v \in M\left(s_{j}\right)$ using less than $i+n_{\text {del }}$ edges:

$$
\begin{aligned}
& C(s):=\left\{u \in V_{\mathrm{r}} \mid \exists v \in M(s): \operatorname{dist}(u, v)<i+n_{\mathrm{del}}\right\}, \\
& \text { where } \operatorname{dist}(u, v) \text { is the length of a shortest walk from } u \text { to } v .
\end{aligned}
$$

Later in this section, we will select $n_{\text {del }}$ to ensure that if an alignment uses more than $n_{\text {del }}$ deletions, its cost must be so high that the heuristic function is trivially admissible.

To compute $C(s)$ efficiently, we can traverse the reference graph backwards from each $v \in M(s)$ by a backward breadth-first-search (BFS).

Heuristic. Let Seed $s_{\geq i}$ be the set of seeds that start at or after position $i$ of the read, formally defined by Seed $s_{\geq i}:=\left\{s_{j}|\lceil i / l\rceil \leq j<|\right.$ Seeds $\left.\mid\right\}$. This allows us to define the number of expected but missing crumbs in state $\langle v, i\rangle$ as misses $\langle v, i\rangle:=\mid\left\{v \notin C(s) \mid s \in\right.$ Seed $\left._{\geq i}\right\} \mid$. Finally, we define the seed heuristic as

$$
\begin{aligned}
& h\langle v, i\rangle=(|q|-i) \cdot \Delta_{\text {match }}+\text { misses }\langle v, i\rangle \cdot \delta_{\text {min }}, \\
& \quad \text { for } \delta_{\text {min }}=\min \left(\Delta_{\text {subst }}-\Delta_{\text {match }}, \Delta_{\text {del }}, \Delta_{\text {ins }}-\Delta_{\text {match }}\right),
\end{aligned}
$$

Intuitively, Eq. (1) reflects that the cost of aligning each remaining letter from $q[i:]$ is at least $\Delta_{\text {match. In }}$ addition, every inexact alignment of a seed induces an additional cost of at least $\delta_{\text {min }}$. Specifically, every substitution costs $\Delta_{\text {subst }}$ but requires one less match; every deletion costs $\Delta_{\text {del }}$; and every insertion costs $\Delta_{\text {subst }}$ but also requires on less match.

We note that $h\langle v, i\rangle$ implicitly also depends on the reference graph $G_{\mathrm{r}}$, the read $q$, the set of seeds, and the edit costs $\Delta$.

In order for an alignment with $n_{\text {del }}$ deletions to have a cost so high that the heuristic function is trivially admissible, we ensure $n_{\text {del }} \cdot \Delta_{\text {del }} \geq h\langle v, i\rangle$ by defining

$$
n_{\mathrm{del}}:=\left\lceil\frac{|q| \cdot \Delta_{\mathrm{match}}+\mid \text { Seeds } \mid \cdot \delta_{\min }}{\Delta_{\mathrm{del}}}\right\rceil .
$$

In Thm. 1, we show that $h\langle v, i\rangle$ is admissible, ensuring that our heuristic yields optimal alignments.

Theorem 1 (Admissibility). The seed heuristic $h\langle v, i\rangle$ is admissible.

Proof. We provide a proof for Thm. 1 in App. A.2. 


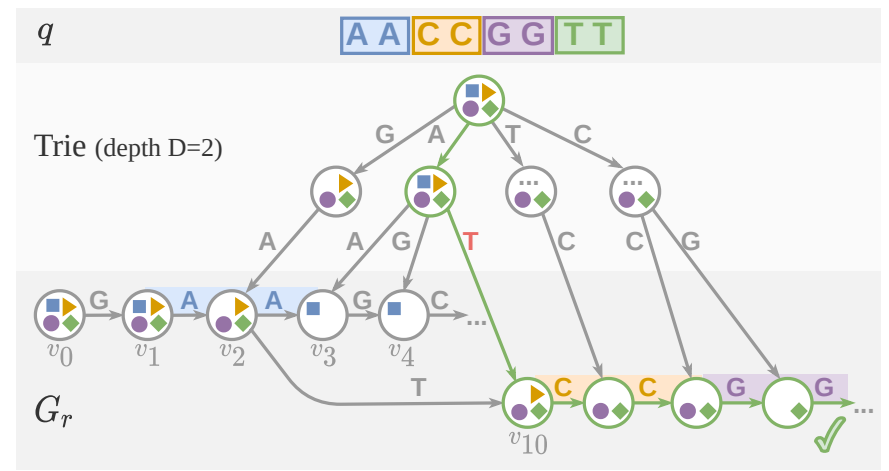

Fig. 3: Reference graph from Fig. 1, extended by a trie of depth $D=2$. For simplicity, the reverse-complement reference graph and parts marked by "..." are omitted.

\subsection{Trie Index}

Considering all nodes $v \in V_{\mathrm{r}}$ as possible starting points for the alignment means that the $\mathrm{A}^{\star}$ algorithm would explore all states of the form $\langle v, 0\rangle$, which immediately induces a high overhead of $\left|V_{\mathrm{r}}\right|$. In line with previous works $[10,12]$, we avoid this overhead by complementing the reference graph with a trie index.

Intuition. Fig. 3 extends the reference graph $G_{r}$ from Fig. 1 with a trie. Here, any path in the reference graph corresponds to a path starting from the trie root (the top-most node in Fig. 3), and vice versa. Thus, in order to find an optimal alignment, it suffices to consider paths starting from the trie root, by using state $\langle$ root, 0$\rangle$ as the only source for the $\mathrm{A}^{\star}$ algorithm.

Since we want to also support aligning reverse-complement reads by starting from the trie root, we build the trie on top of the union of the original reference graph and its reverse-complement.

For a more thorough introduction to the trie and its construction, see [10]. Importantly, our placement of crumbs (§3.2) generalizes directly to reference graphs extended with a trie (see also Fig. 3).

Using Trie to Find Seed Matches. We can also exploit the trie to efficiently locate all matches $M(s)$ of a given seed $s$. In order to find all nodes where a seed match begins, we align (without errors) $\bar{s}$, the reverse-complement of $s$. To this end, we follow all paths spelling $\bar{s}$ starting from the root - the final nodes of these paths then correspond to nodes in $M(s)$. We ensure that the seed length $|s|$ is not shorter than the trie depth $D$, so that matching all letters in $\bar{s}$ ensures that we end up in the reference graph.

Skipping Crumbs in Trie. Generally, we aim to place as few crumbs as possible, in order to both reduce precomputation time and avoid misleading the $\mathrm{A}^{\star}$ algorithm by unnecessary crumbs. In the following, we introduce an optimization to avoid placing crumbs on trie nodes that are "too close" to the match of their corresponding seed so they cannot lead to an optimal alignment.

Specifically, when traversing the reference graph backwards to place crumbs for a match of seed $s$ starting at node $w$, we may "climb" from a reference graph node $u$ to a trie node $u^{\prime}$ backwards through an edge that otherwise leads from the trie to the reference. Assuming $s$ starts at position $i$ in the read, we have already established that we can only consider nodes $u$ that can reach $w$ with less than $i+n_{\text {del }}$ edges (see §3.2). Here, we observe that it is sufficient to only climb into the trie from nodes $u$ that can reach $w$ using more than $i-n_{\text {ins }}-D$ edges, for

$$
n_{\mathrm{ins}}:=\left\lceil\frac{|q| \cdot \Delta_{\mathrm{match}}+n \cdot \delta_{\min }}{\Delta_{\mathrm{ins}}}\right\rceil \text {. }
$$

We define $n_{\text {ins }}$ analogously to $n_{\text {del }}$ to ensure that $n_{\text {ins }}$ insertions will induce a cost that is always higher than $h\langle u, i\rangle$. We note that we can only avoid climbing into the trie if all paths from $u$ to $w$ are too short, in particular the longest one.

The following Lemma 1 shows that this optimization preserves optimality. 
Lemma 1 (Admissibility when Skipping Crumbs) The seed heuristic remains admissible when crumbs are skipped in the trie.

Proof. We provide a proof for Lemma 1 in App. A.2.

In order to efficiently identify all nodes $u$ that can reach $w$ by using more than $i-D-n_{\text {ins }}$ edges (among all nodes at a backward-distance at most $i+n_{\text {del }}$ from $w$ ), we use topological sorting: considering only nodes at a backward-distance at most $i+n_{\text {del }}$ from $w$, the length of a longest path from a node $v$ to $w$ is (i) $\infty$ if $v$ lies on a cycle and (ii) computable from nodes closer to $w$ otherwise.

\section{Evaluations}

In the following, we demonstrate that our approach is faster than existing aligners due to its superior scaling. Specifically, we address the following research questions in relation to existing optimal aligners:

Q1 What speedup can seed heuristic achieve?

Q2 How does seed heuristic scale with reference size?

Q3 How does seed heuristic scale with read length?

The modes of operation which we analyze include both short (Illumina) and long (HiFi) reads to be aligned on on both linear and graph references.

Existing Aligners. To this end, we compare our seed heuristic to prefix heuristic, GraphAligner, PaSGAL, and VARgas (both heuristics are implemented in AStarix). We note that we do not compare to VG [1] and HGA [9]. The optimal alignment functionality of VG is meant for debugging purposes and has been shown to be inferior to other aligners [9, Tab. 4], and HGA makes use of parallelization and a GPU but has been shown to be superseded in the single CPU setting of our evaluations [9, Fig. 9].

\subsection{Setting}

All experiments were executed on a commodity machine with an Intel Core i7-6700 CPU @ 3.40GHz processor, using a memory limit of $20 \mathrm{~GB}$ and a single thread. We note that while multiple tools support parallelization when aligning a single read, all tools can be trivially parallelized to align multiple reads in parallel. PASGAL and VARGAS are compiled with AVX2 support.

Metrics. We report the performance of aligners in terms of runtime per one thousand aligned base pairs [s/kbp]. Since we measured runtime end-to-end (including loading reference graphs and reads from disk, and building the trie index for ASTARIX), we ensured that alignment time dominates the total runtime by providing sufficiently many reads to each aligner. In order to prevent excessive runtimes for slower tools, we used a different number of reads for each tool and explicitly report them for each experiment.

We note that since shortest path approaches skip considerable parts of the computation performed by aligners based on dynamic programming, the commonly used Giga Cell Updates Per Second (GCUPS) metric is not adequate for measuring performance in our context.

To measure used memory, we report max_rss (Maximum Resident Set Size) according to Snakemake ${ }^{2}$.

We do not report accuracy or number of unaligned reads, as all evaluated tools align all reads with guaranteed optimality according to edit distance. We note that VARGAS reports a warning that some of its alignments are not optimal — we ignore this warning and focus on its performance.

Data. We aligned reads to two different reference graphs: a linear E. coli genome (strain: K-12 substr. MG1655, ASM584v2) [13], with length of $4641652 \mathrm{bp}$ (approx. 4.7Mbp), and a variant graph with the Major Histocompatibility Complex (MHC), of size 5318019bp (approx. 5Mbp) (taken from the evaluations of PASGAL) [7]. Additionally, we extracted a path from MHC in order to create a linear reference MHClinear of length $4956648 \mathrm{bp}$ which covers approx. $93 \%$ of the original graph. Because of input graph format restrictions, we execute GraphAligner, VARgas and PASGAL only on linear references in FASTA format (E. coli and the MHC-linear), while we execute the seed heuristic and the prefix heuristic on the original

\footnotetext{
${ }^{2}$ https://snakemake.readthedocs.io/en/stable/
} 
Table 1: Runtime and memory comparison of optimal aligners. Simulated Illumina and HiFi reads are aligned to linear E. coli and graph MHC references. The runtime of seed heuristic is expressed as absolute time per aligned kbp, while the other aligners are compared to seed heuristic. Additionally, the fraction of explored states is shown for seed heuristic and prefix heuristic.

\begin{tabular}{|c|c|c|c|c|c|}
\hline \multirow[b]{2}{*}{ Tool } & \multicolumn{2}{|c|}{ Illumina } & \multicolumn{2}{|c|}{ HiFi } & \\
\hline & E. coli & $\mathrm{MHC}$ & E. coli & $\mathrm{MHC}$ & \\
\hline $\begin{array}{c}\text { Seeds heuristic } \\
\text { (this work) }\end{array}$ & $\begin{array}{r}0.019 \\
2.4 \\
99.99969\end{array}$ & $\begin{array}{r}0.041 \\
2.6 \\
9.9981\end{array}$ & $\begin{array}{c}0.001 \\
2.4 \\
99.99899\end{array}$ & $\begin{array}{r}0.002 \\
1.7 \\
9.9984\end{array}$ & $\begin{array}{l}\text { s/kbp } \\
\text { GB } \\
\% \text { skipped states }\end{array}$ \\
\hline Prefix heuristic & $\begin{array}{r}269 x \\
7.7 \\
99.95019\end{array}$ & $\begin{array}{r}180 x \\
9.6 \\
9.9501\end{array}$ & $\begin{array}{r}\mathrm{n} / \mathrm{a} \\
>20 \\
\mathrm{n} / \mathrm{a}\end{array}$ & $\begin{array}{r}\mathrm{n} / \mathrm{a} \\
>20 \\
\mathrm{n} / \mathrm{a}\end{array}$ & x slowdown \\
\hline GraphAligner & $\begin{array}{r}424 x \\
0.2\end{array}$ & $\begin{array}{r}212 x \\
0.2\end{array}$ & $\begin{array}{r}118 x \\
3.6\end{array}$ & $\begin{array}{r}64 x \\
3.4\end{array}$ & \\
\hline VARGAS & $\begin{array}{l}133 x \\
<0.1\end{array}$ & $\begin{array}{r}67 x \\
<0.1\end{array}$ & $\begin{array}{r}1413 x \\
7.3\end{array}$ & $\begin{array}{r}705 x \\
7.3\end{array}$ & \\
\hline PASGAL & $\begin{array}{r}263 x \\
0.6\end{array}$ & $\begin{array}{r}130 x \\
0.6\end{array}$ & $\begin{array}{r}1367 x \\
0.6\end{array}$ & $\begin{array}{r}736 x \\
0.6\end{array}$ & \\
\hline
\end{tabular}

references (E. coli and MHC). This yields an underestimation of the speedup of seed heuristic, as we expect the performance on MHC-linear to be strictly better than on the whole MHC graph.

To generate both short Illumina and long HiFi reads, we relied on two tools. We generated short singleend 200bp Illumina MSv3 reads using ART simulator [14]. We generated long HiFi reads using the script RANDOMREADS. $\mathrm{SH}^{3}$ with sequencing lengths $5-25 \mathrm{kbp}$ and error rates $0.3 \%$, which are typical for HiFi reads.

Commands. We provide the versions and commands used to run all aligners and read simulators in App. A.3.

\subsection{Implementation and Parameter Choices}

Implementation. The seed heuristic and prefix heuristic reuse the same free and open source $\mathrm{C}++$ codebase of the ASTARIX aligner [10]. It includes a simple implementation of a graph and trie data structure which is not optimized for memory usage. In order to easily align reverse complement reads, the reverse complement of the graph is stored alongside its straight version. The shortest path algorithm only constructs explored states explicitly, so most states remain implicitly defined and do not cause computational burden.

Both heuristics benefit from a default optimization in ASTARIX called greedy matching [10, Section 4.2] which allows to skip adding a state to the $\mathrm{A}^{\star}$ queue when only one edge is outgoing from a state and the upcoming read and reference letters match.

Parameters. We execute ASTARIX with edit costs corresponding to the specific sequencing technology: $\Delta=(0,1,5,5)$ for Illumina reads and $\Delta=(0,1,1,1)$ for HiFi reads. We set the trie depth to $D=14$. We parametrize the prefix heuristic with the default lookup depth $d=5$. We parametrize the seed heuristic with seed length $l=25$ for Illumina reads and $l=150$ for HiFi reads. As the performance of DP-based tools is independent of edit costs, we are using the respective default edits costs when running GrAPHALIGNER, PASGAL and VARGAS.

\subsection{Q1: Speedup of the Seed Heuristic}

Table 1 shows that seed heuristic achieves a speedup of at least 60 times compared to all considered aligners, across all regimes of operation: both Illumina and HiFi reads aligned on E. coli and MHC references.

\footnotetext{
${ }^{3}$ https://github.com/BioInfoTools/BBMap/blob/master/sh/randomreads.sh
} 

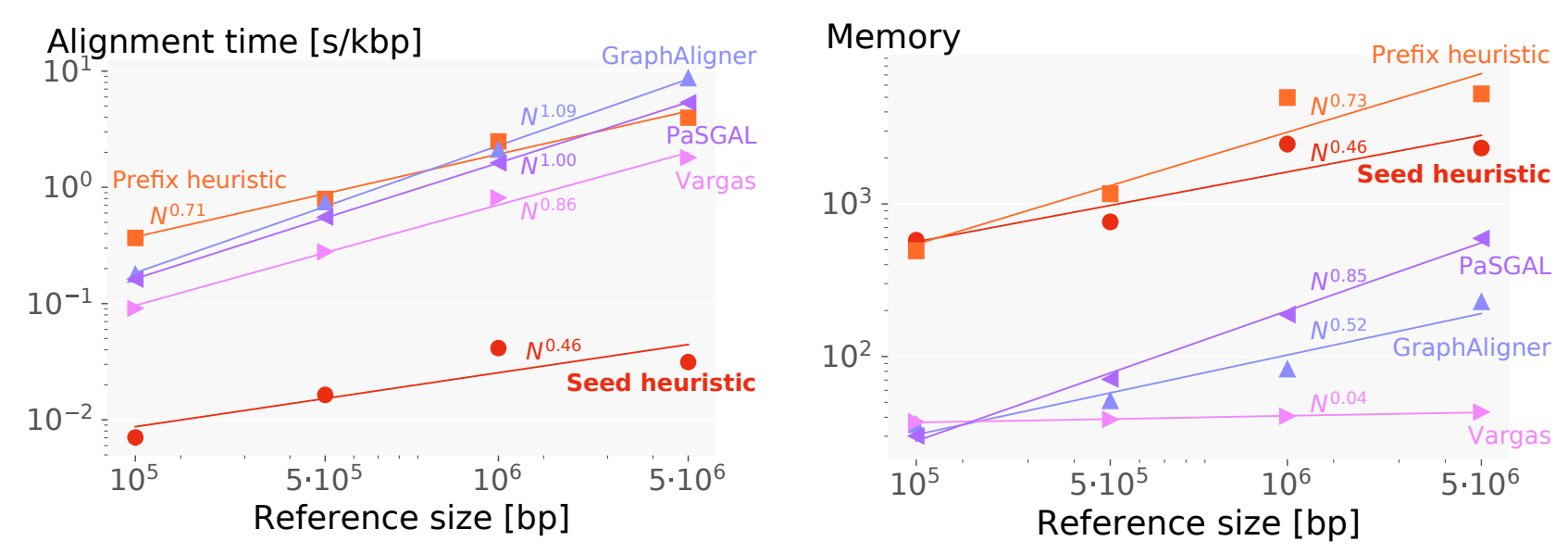

Fig. 4: Performance degradation with reference size for Illumina reads. Log-log plots of total alignment time (left) and memory usage (right) show the scaling difference between aligners.
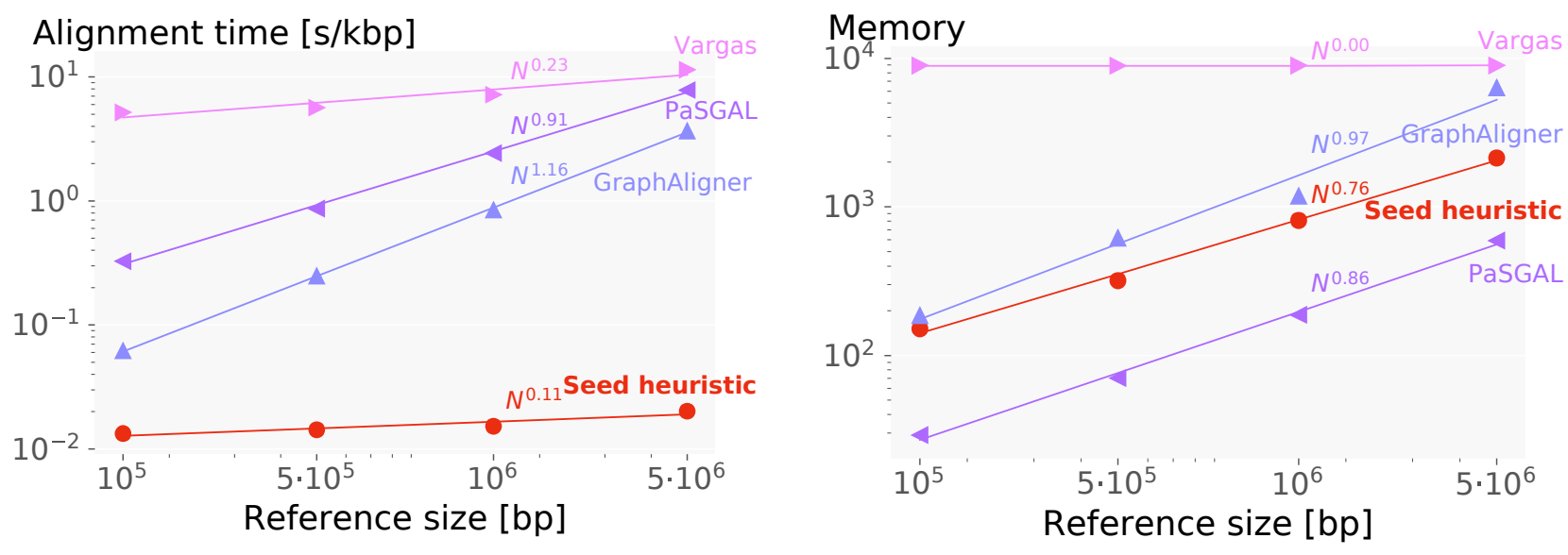

Fig. 5: Performance degradation with reference size for HiFi reads. Log-log plots of total alignment time (left) and memory usage (right) show the scaling difference between aligners. Linear best fits correspond to polynomials of varying degree.

In the Illumina experiments, seed heuristic is given 100k reads, while the other tools are given 1000 reads. In the HiFi experiments, seed heuristic is given reads that cover the reference 10 times, and the other tools are given reads of coverage 0.1 .

The core reason for the speedup of the seed heuristic is that on all four experiments, it skips $\geq 99.99 \%$ of the $N m$ states computed by the DP approaches of GraphAligner, PaSGAL and VArgas. This fraction accounts for both the explored states during the $\mathrm{A}^{\star}$ algorithm, and for the number of crumbs added to nodes during the precomputation for each read.

The prefix heuristic exceeded the available memory on HiFi reads, as it is not designed for long reads.

\subsection{Q2: Scaling with Reference size}

In order to study the scaling of the aligners in terms of the reference size, we extracted prefixes of increasing length from MHC-linear. We then generated reads from each prefix, and ran all tools on all prefixes with the corresponding reads.

Illumina reads. Fig. 4 shows the runtime scaling and memory usage for Illumina reads. The seed heuristic was provided with $10 \mathrm{k}$ reads, while other tools were provided with $1 \mathrm{k}$ reads. The runtime of GrAPHALIGNER, 

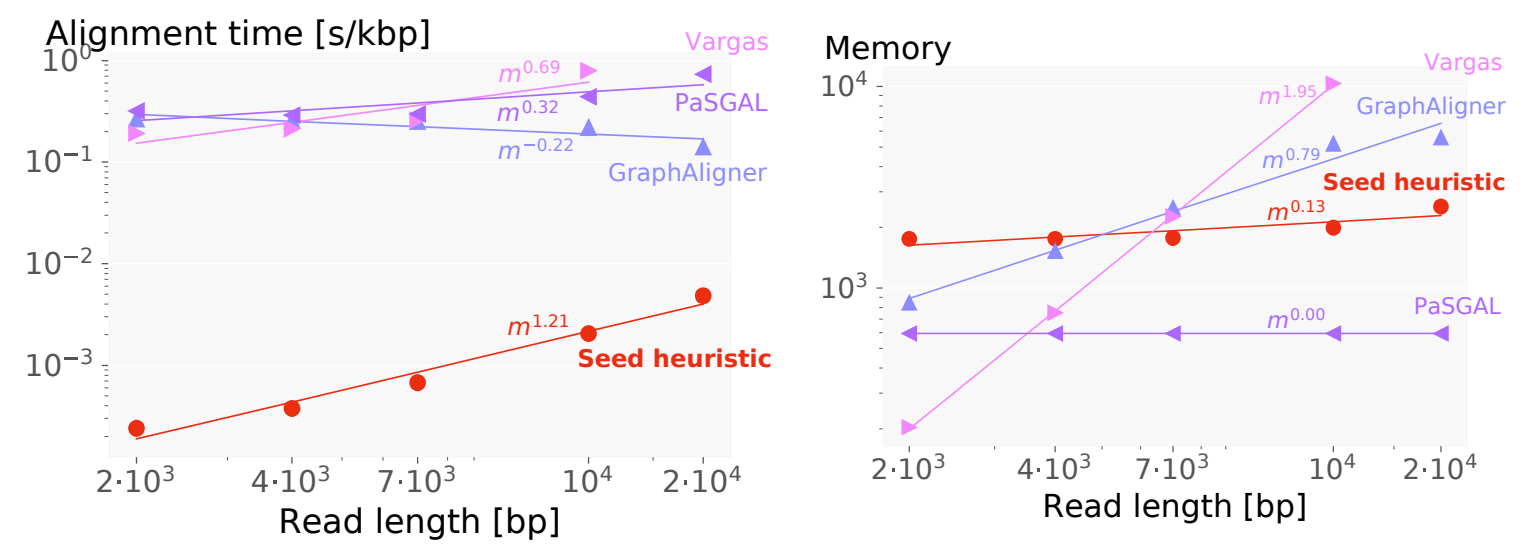

Fig. 6: Performance degradation with HiFi read length. Log-log plots of total alignment time (left) and memory usage (right) show the scaling difference between aligners.

PASGAL and VARGAS grow approximately linearly with the reference length, whereas the runtime of the seed heuristic grows roughly with $\sqrt[2]{N}$, where $N$ is the reference size. Even on relatively small graphs like MHC, the speedup of the seed heuristic reaches 200 times. Note that the scaling of the prefix heuristic is substantially worse than the seed heuristic since the $200 \mathrm{bp}$ reads are outside of its operational capabilities.

HiFi reads. Fig. 5 shows the runtime scaling and memory usage for HiFi reads. The respective total lengths of all aligned reads are $5 \mathrm{Mbp}$ for the seed heuristic, $500 \mathrm{kbp}$ for GRAPHALIGNER, and 100kbp for VARGAS and PASGAL. We do not show the prefix heuristic, since it explores too many states and runs out of memory. Crucially, we observe that the runtime of the seed heuristic is almost independent of the reference size, growing as $N^{0.11}$. We believe this improved trend compared to short reads is because the seed heuristic obtains better guidance on long reads, as it can leverage information from the whole read.

For both Illumina and HiFi reads, we observe near-linear scaling for PASGAL and GraPhAligner as expected from the theoretical $\mathcal{O}(\mathrm{Nm})$ runtime of the DP approaches. We conjecture that the runtime of VARGAS for long reads is dominated by the dependence from the read length, which is why on HiFi reads we observe better than linear runtime dependency on $N$ but very large runtime. The current alignment bottleneck of ASTARIX-SEEDS is its memory usage, which is distributed between remembering crumbs and holding a queue of explored states.

\subsection{Q3: Scaling with Read length}

Fig. 6 shows the runtime and memory scaling with increasing length of aligned HiFi reads on MHC reference. Here we used reads with a total length of $100 \mathrm{Mbp}$ for the seed heuristic and $2 \mathrm{Mbp}$ for all other aligners.

The scaling of the seed heuristic in terms of read length is slightly worse than that of other aligners. However, this is compensated by its superior scaling in terms of reference size (see §4.4), leading to an overall better absolute runtime. We note that the memory usage of seed heuristic does not heavily depend on the read length and for reads longer than $10 \mathrm{kbp}$, it is superior to GRAPHALIGNER and VARGAS.

\section{Conclusion}

We have presented an optimal read aligner based on the $\mathrm{A}^{\star}$ algorithm instantiated with a novel seed heuristic which guides the search by placing crumbs on nodes that lead towards optimal alignments even for long reads.

A remaining challenge is designing a heuristic function able to handle not only long but also noisier reads, such as the uncorrected PacBio reads that may reach $20 \%$ of mistakes. Memory usage is the current bottleneck for both the ASTARIX tool and the seed heuristic: larger references incur large trie indexes, as well as more crumbs and explored states. 


\section{References}

1. E. Garrison, J. Sirén, A. M. Novak, G. Hickey, J. M. Eizenga, E. T. Dawson, W. Jones, S. Garg, C. Markello, M. F. Lin, B. Paten, and R. Durbin, "Variation graph toolkit improves read mapping by representing genetic variation in the reference," Nature Biotechnology, 2018.

2. S. F. Altschul, W. Gish, W. Miller, E. W. Myers, and D. J. Lipman, "Basic local alignment search tool," Journal of Molecular Biology, 1990.

3. B. Langmead and S. L. Salzberg, "Fast gapped-read alignment with Bowtie 2," Nature Methods, 2012.

4. H. Li and R. Durbin, "Fast and accurate short read alignment with Burrows-Wheeler transform," Bioinformatics (Oxford, England), 2009.

5. M. Equi, R. Grossi, V. Mäkinen, A. Tomescu, et al., "On the complexity of string matching for graphs," in 46th International Colloquium on Automata, Languages, and Programming (ICALP 2019), Schloss Dagstuhl-LeibnizZentrum für Informatik, 2019.

6. C. A. Darby, R. Gaddipati, M. C. Schatz, and B. Langmead, "Vargas: heuristic-free alignment for assessing linear and graph read aligners," Bioinformatics, vol. 36, no. 12, pp. 3712-3718, 2020.

7. C. Jain, S. Misra, H. Zhang, A. Dilthey, and S. Aluru, "Accelerating Sequence Alignment to Graphs," in International Parallel and Distributed Processing Symposium (IPDPS), 2019. ISSN: 1530-2075.

8. M. Rautiainen, V. Mäkinen, and T. Marschall, "Bit-parallel sequence-to-graph alignment," Bioinformatics, 2019.

9. Z. Feng and Q. Luo, "Accelerating sequence-to-graph alignment on heterogeneous processors," in 50th International Conference on Parallel Processing, pp. 1-10, 2021.

10. P. Ivanov, B. Bichsel, H. Mustafa, A. Kahles, G. Rätsch, and M. T. Vechev, "AStarix: Fast and Optimal Sequenceto-Graph Alignment," in RECOMB 2020, 2020.

11. M. Rautiainen and T. Marschall, "Aligning sequences to general graphs in $O(V+m E)$ time," preprint, Bioinformatics, Nov. 2017.

12. G. Dox and J. Fostier, "Efficient algorithms for pairwise sequence alignment on graphs," Master's thesis, Ghent university, 2018.

13. K. L. Howe, B. Contreras-Moreira, N. De Silva, G. Maslen, W. Akanni, J. Allen, J. Alvarez-Jarreta, M. Barba, D. M. Bolser, L. Cambell, et al., "Ensembl Genomes 2020-enabling non-vertebrate genomic research," Nucleic Acids Research, 2020.

14. W. Huang, L. Li, J. R. Myers, and G. T. Marth, "ART: a next-generation sequencing read simulator," Bioinformatics, vol. 28, no. 4, pp. 593-594, 2011. 


\section{A Appendix}

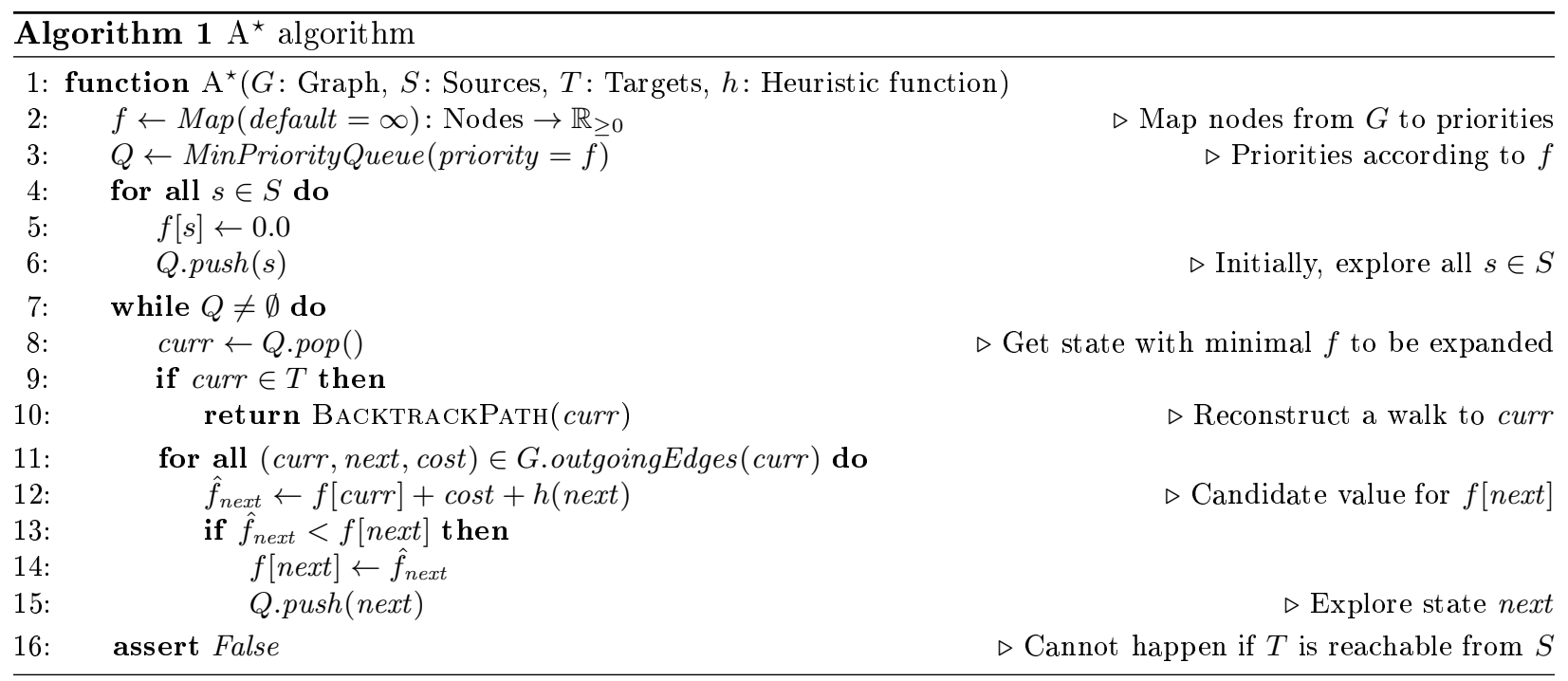

\section{A.1 $\quad \mathbf{A}^{\star}$ Algorithm}

Algorithm 1 shows an implementation of the $\mathrm{A}^{\star}$ algorithm, taken from [10, §A.1]. We omit the implementation of BACKTRACKPath for simplicity.

\section{A.2 Proofs}

In the following, we provide proofs for Thm. 1 and Lemma 1, restated here for convenience.

Theorem 1 (Admissibility). The seed heuristic $h\langle v, i\rangle$ is admissible.

Proof. Let $A$ be an optimal alignment of $q[i$ : $]$ starting from $v \in G_{\mathrm{r}}$. We will prove that the cost of $A$ is at least $h\langle v, i\rangle$.

If $A$ contains at least $n_{\text {del }}$ deletions, its cost is at least $n_{\text {del }} \cdot \Delta_{\text {del }}$, which is at least $|q| \cdot \Delta_{\text {match }}+n \cdot \delta_{\min }$ by plugging in $n_{\text {del }}$ from Eq. (3). This is an upper bound for $h\langle v, i\rangle$, which we observe after maximizing $h\langle v, i\rangle$ by substituting $i=1$ and misses $=n$ into Eq. (1), which concludes the proof in this case.

Otherwise, $A$ contains less than $n_{\text {del }}$ deletions. If we interpret $A$ as a path in $G_{a}^{q}$, we first observe that $A$ must spell $q\left[i:\right.$. Thus, $A$ must in particular also contain all seeds $s_{j} \in$ Seeds $s_{\geq i}$ as substrings. We then split $A$ into subalignments $A_{-1}, A_{0}, \ldots, A_{p}$, selected such that $A_{0}, \ldots, A_{p-1}$ spell the seeds $s_{j} \in$ Seed $s_{\geq i}$, and $A_{-1}$ and $A_{p}$ spell the prefix and suffix of $q[i$ : $]$ which do not cover any full seed.

This ensures that we can compute a lower bound on the cost of $A$ as follows:

$$
\begin{aligned}
\operatorname{cost}(A) & =\sum_{k=-1}^{p} \operatorname{cost}\left(A_{k}\right) \\
& \geq \sum_{k=-1}^{p}\left|\sigma\left(A_{k}\right)\right| \cdot \Delta_{\text {match }}+\sum_{k=0}^{p-1}\left\{\begin{array}{ll}
0 & \text { if } v \in C\left(s_{\lceil i / l\rceil+k}\right) \\
\delta_{\text {min }} & \text { if } v \notin C\left(s_{\lceil i / l\rceil+k}\right)
\end{array}\right\} \\
& =(|q|-i) \cdot \Delta_{\text {match }}+\mid\left\{v \notin C(s) \mid s \in \text { Seed } s_{\geq i}\right\} \mid \cdot \delta_{\text {min }} \\
& =h\langle v, i\rangle
\end{aligned}
$$

Here, Eq. (5) follows from our decomposition of $A$. If we ignore the right-hand side in Eq. (6) (right of "+"), the inequality follows because matching all letters is the cheapest method to align any string. The 
right-hand side follows from a more precise analysis for subalignments $A_{k}$ that spell a seed $s_{\lceil i / l\rceil+k}$ without a corresponding crumb in $v$. The absence of such a crumb indicates that no exact match of $s_{\lceil i / l\rceil+k}$ in $G_{\mathrm{r}}$ can be reached within less than $i+n_{\text {del }}$ steps from $v$. However, because $A$ contains less than $n_{\text {del }}$ deletions, $A_{k}$ must start within less than $i+n_{\text {del }}$ steps from $v$. Thus, $A_{k}$ does not align $s_{\lceil i / l\rceil+k}$ exactly, meaning that it introduces a cost of at least $\delta_{\min }$.

Eq. (7) follows from observing that $A_{-1}, \ldots, A_{p}$ have a total length of $|q|-i$, and observing that the righthand sum adds up $\delta_{\min }$ for every expected but missing crumb. Finally, Eq. (8) follows from our definition of $h\langle v, i\rangle$, concluding the proof.

Lemma 1 (Admissibility when Skipping Crumbs) The seed heuristic remains admissible when crumbs are skipped in the trie.

Proof. Consider a reference graph with a match of seed $s$ starting in node $w$. Now, consider a node $v$ that cannot reach $w$ using more than $i-D-n_{\text {ins }}$ edges. We can then show that a trie node $v^{\prime}$ with a path to $v$ does not require a crumb for the match of $s$ in node $w$.

Specifically, any path from root through nodes $v^{\prime}$ and $v$ to node $w$ has total length $i-n_{\text {ins }}$. Thus, matching $s$ at $w$ requires at least $n_{\text {ins }}$ insertions. Hence, the cost of such a path is at least $n_{\text {ins }} \cdot \Delta_{\text {ins }}=|q| \cdot \Delta_{\text {match }}+n \cdot \delta_{\min }$. Observing that this is an upper bound for $h\langle v, i\rangle$ concludes the proof.

\title{
A.3 Versions, commands, parameters for running all evaluated approaches
}

In the following, we provide details on how we executed the newest versions of the tools discussed in $\S 4$ :

\section{Running AStarix.}

Obtained from https://github.com/eth-sri/astarix seed heuristic

Command astarix align-optimal -D 14 -a astar-seeds -seeds_len $l$ - $f$ reads.fq - $g$ graph.gfa >output

\section{prefix heuristic}

Command astarix align-optimal -D 14 -a astar-prefix -d 5 - $f$ reads. fq -g graph.gfa >output

For aligning Illumina reads, astarix is used with additional -M 0 -S 1 - - G 5 and for HiFi reads with -M

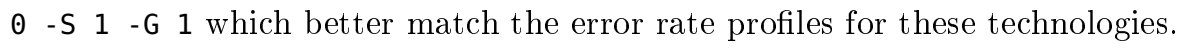

\author{
Running other tools. \\ VARGAS \\ Obtained from https://github.com/langmead-lab/vargas (v0.2, commit \\ blad5d9) \\ Command vargas align -g graph.gdef $-U$ reads.fq - ete \\ Comment - -ete stands for end to end alignment; default is 1 thread \\ PASGAL \\ Obtained from \\ Command \\ https://github.com/ParBLiSS/PaSGAL (commit 9948629) \\ Comment \\ PaSGAL - $q$ reads.fq $-r$ graph.vg $-m$ vg - o output - $t 1$ \\ Graph Aligner \\ Obtained from https://github.com/maickrau/GraphAligner (v1.0.13, commit \\ Compiled with AVX2. \\ 02c8e26) \\ Command \\ GraphAligner -seeds-first-full-rows 64 -b 10000 -t 1 - $f$ \\ reads.fq -g graph.gfa -a alignments.gaf >output (commit \\ 9948629) \\ Comment \\ - seeds - first - full - rows forces the search from all possible ref- \\ erence positions instead of using seeds; -b 10000 sets a high \\ alignment bandwidth; these two parameters are necessary for \\ an optimal alignment according to the author and developer \\ of the tool.
}


bioRxiv preprint doi: https://doi.org/10.1101/2021.11.05.467453; this version posted November 8, 2021. The copyright holder for this preprint (which was not certified by peer review) is the author/funder, who has granted bioRxiv a license to display the preprint in perpetuity. It is made available under aCC-BY 4.0 International license.

Simulating reads.

Illumina

HiFi

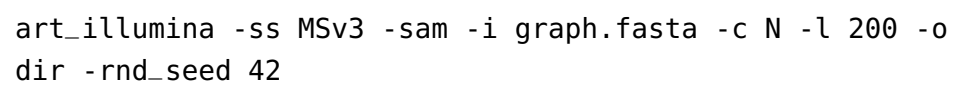

randomreads. $\mathrm{sh}-\mathrm{Xm \times 1g}$ build=1 ow=t seed=1 ref=graph. fa

illuminanames $=\mathrm{t}$ addslash $=\mathrm{t}$ pacbio $=\mathrm{t}$ pbmin $=0.003$

pbmax $=0.003$ paired $=f$ gaussianlength $=t$ minlength $=5000$

midlength=13000 maxlen=25000 out=reads. fq

Comment BBMapcoverage, https://github.com/BioInfoTools/BBMap/blob/master/sh/randomreads.sh (commit: a9ceda0)

\section{A.4 Notations}

Table 2 summarizes the notational conventions used in this work.

Table 2: Notational conventions.

\begin{tabular}{|c|c|}
\hline Object & Notation \\
\hline Queries & $Q=\left\{q_{i} \mid q_{i} \in \Sigma^{m}\right\}$ \\
\hline Read & $q \in Q$ \\
\hline Length & $m:=|q| \in \mathbb{N}$ \\
\hline Position in read & $q[i] \in \Sigma, i \in\{1, \ldots, m\}$ \\
\hline Reference graph & $G_{\mathrm{r}}=\left(V_{\mathrm{r}}, E_{\mathrm{r}}\right)$ \\
\hline Size & $\left|G_{\mathrm{r}}\right|:=\left|V_{\mathrm{r}}\right|+\left|E_{\mathrm{r}}\right| \in \mathbb{N}$ \\
\hline Nodes & $u, v \in V_{\mathrm{r}}, n:=\left|V_{\mathrm{r}}\right| \in \mathbb{N}$ \\
\hline Edges & $e \in E_{\mathrm{r}}:=V_{\mathrm{r}} \times V_{\mathrm{r}} \times \Sigma$ \\
\hline Edge letter & $\ell \in \Sigma$ \\
\hline \multicolumn{2}{|c|}{ Reference graph with a trie $G_{\mathrm{r}}^{+}=\left(V_{\mathrm{r}}^{+}, E_{\mathrm{r}}^{+}\right)$} \\
\hline Trie depth & $D \in \mathbb{N}_{>0}$ \\
\hline Alignment graph & $G_{\mathrm{a}}^{q}=\left(V_{\mathrm{a}}^{q}, E_{\mathrm{a}}^{q}\right)$ \\
\hline Size & $N:=\left|V_{\mathrm{a}}^{q}\right| \in \mathbb{N}$ \\
\hline State & $\langle u, i\rangle \in V_{\mathrm{a}}^{q}:=V \times\{0, \ldots, m\}$ \\
\hline Edges & $(\langle u, i\rangle,\langle v, j\rangle, \ell, w) \in E_{\mathrm{a}}^{q} \subseteq V_{\mathrm{a}}^{q} \times V_{\mathrm{a}}^{q} \times \Sigma_{\varepsilon} \times \mathbb{R}_{\geq 0}$, where $\Sigma_{\varepsilon}=\Sigma \cup\{\varepsilon\}$ \\
\hline Edge cost & $w \in \mathbb{R}_{\geq 0}$ \\
\hline Alignment & $\pi \in E_{\mathrm{e}}^{\bar{*}}$ and $\sigma(\pi)=q$ \\
\hline Alignment cost & $\operatorname{cost}(\pi) \in \mathbb{R}_{\geq 0}$ \\
\hline Seed heuristic & $h\langle u, i\rangle$ \\
\hline Maximal number of deletions & $n_{\mathrm{del}}$ \\
\hline Seed length & $l$ \\
\hline In all graphs & $G(V, E) \in\left\{G_{\mathrm{r}}, G_{\mathrm{e}}, G_{\mathrm{a}}^{q}\right\}$ \\
\hline Walk & $\pi \in G: \pi \in E^{*}$ \\
\hline Walk spelling & $\sigma(\pi) \in \Sigma^{*}$ \\
\hline Path & A walk without repeating nodes \\
\hline$\overline{\mathbf{A}^{\star}}$ & $A^{\star}(G, S, T, h)$ \\
\hline Graph & $G=(V, E)$ \\
\hline Nodes & $u, v \in V$ \\
\hline Edges & $e \in E \subseteq V \times V \times \mathbb{R}_{\geq 0}$ \\
\hline Source states & $S \subseteq V$ \\
\hline Target states & $T \subseteq V$ \\
\hline Heuristic function & $h(u \in V): V \rightarrow \mathbb{R}_{\geq 0}$ \\
\hline Minimum cost to a target & $h^{*}(u)$ \\
\hline Optimistic & $h(u) \leq \min _{\pi} \operatorname{cost}(\pi), \pi: \pi$ starts from $u$ \\
\hline Explored state & A state pushed to the queue of Algorithm 1 \\
\hline Expanded state & A state popped from the queue of Algorithm 1 \\
\hline
\end{tabular}

